\title{
A case of enteroviral meningoencephalitis presenting as rapidly progressive dementia
}

\author{
Victor Valcour ${ }^{*}$, Aissa Haman, Susannah Cornes, Carson Lawall, Andrew T Parsa, Carol \\ Glaser, Shigeo Yagi, Tarik Tihan, Julu Bhatnagar, and Michael Geschwind \\ $V$ Valcour is a Fellow in Neurobehavior, A Haman is a Research Analyst, $S$ Cornes is a Clinical \\ Instructor of Neurology, C Lawall is a Resident in Neurology, and M Geschwind is an Assistant \\ Professor of Neurology, in the Memory and Aging Center, Department of Neurology, AT Parsa is \\ an Associate Professor of Neurological Surgery in the Department of Neurosurgery, and T Tihan is \\ an Associate Professor of Pathology in the Department of Pathology, all at the University of \\ California, San Francisco, CA. C Glaser is Principal Investigator for the California Encephalitis \\ Project and Chief of the Viral and Rickettsial Disease Laboratory, and S Yagi is a Research Scientist \\ in the Viral and Rickettsial Disease Laboratory, in the Division of Communicable Disease Control, \\ California Department of Public Health, Richmond, CA. J Bhatnagar is a Microbiologist in the \\ Infectious Disease Pathology Branch, Centers for Disease Control and Prevention, Atlanta, GA, \\ USA
}

\section{SUMMARY}

Background-A 70-year-old immunocompetent male presented to a memory disorders clinic with a 7-month illness that had begun with somatic complaints including transient right temporal head pain, left buttock pain, and right conjunctival injection. About 3 months after the first signs of illness, the patient had begun to develop insidious cognitive and behavioral decline, which progressed most rapidly in the 2 months before presentation. An assessment completed during hospitalization for intermittent fevers and confusion had not revealed an infectious etiology, although mild pleocytosis in the cerebrospinal fluid had been noted. Upon presentation to the memory disorders clinic, the patient was disoriented, distractible, laughed at inappropriate moments, and followed only one-step commands. He had hypophonic speech and had mildly increased axial tone. He scored 5 out of 30 on the Mini Mental State Examination and was admitted for expedited evaluation.

Investigations-Physical examination, brain MRI, electroencephalogram, lumbar puncture, autoimmune and paraneoplastic testing, cerebral angiogram, cerebrospinal fluid analysis, enterovirus group-specific reverse transcriptase polymerase chain reaction assay, and RNA sequencing in brain biopsy samples.

Diagnosis-Enteroviral meningoencephalitis.

Management-Intravenous steroids with oral taper and intravenous immunoglobulin.

\section{Keywords}

dementia; echovirus; encephalitis; enterovirus; meningitis

\footnotetext{
*Correspondence Memory and Aging Center, Department of Neurology, University of California at San Francisco, 350 Parnassus Avenue, Suite 706, San Francisco, CA 94143, USA vvalcour@memory.ucsf.edu.

Vanderbilt Continuing Medical Education online

This article offers the opportunity to earn one Category 1 credit toward the AMA Physician's Recognition Award.

Competing interests

The authors declared no competing interests.
} 


\section{THE CASE}

A 70-year-old male presented to a memory disorders clinic with a 7-month illness that had begun insidiously with somatic complaints and progressed to rapid cognitive decline. The patient's first symptoms had included intermittent right temporal head pain with radiation posteriorly and blurred vision. He then reported transient left buttock pain with calf numbness and, on a separate occasion, low back pain. Early in the course of the illness, he had developed right conjunctival injection, which was treated successfully with steroid drops.

The patient's wife had noted slowly progressive confusion that began insidiously in a fluctuating manner and included repetitive questioning and mild difficulty with household orientation. The patient had been hospitalized for nonspecific chest pain and hyponatremia (serum sodium level $117 \mathrm{mmol} / \mathrm{l}$ ), which resolved and did not recur following discontinuation of a thiazide diuretic. His cognitive symptoms had progressed over the next few months, resulting in an inability to recognize people, and the patient would sometimes get lost while driving locally. Insomnia, flattened affect and hypomimia led to a diagnosis of depression, but the patient's symptoms did not improve with antidepressant therapy. Tremors and recurrent headaches emerged. The patient developed mild disinhibition and began compulsively checking doors to ensure they were locked. He believed that deceased relatives were still alive and reported hearing voices. His speech became rambling and tangential with decreased comprehension and intermittent echolalia. Shuffling was noted in his gait. Over the course of the illness, he lost $20 \mathrm{lbs}(9 \mathrm{~kg})$ and became dependent on others for basic care.

One month before presentation to the memory disorders clinic, the patient had developed recurrent low grade fever and headaches and had been admitted to a community hospital. Initial analysis of his cerebrospinal fluid (CSF) revealed a white blood cell count of $7 \times 10^{6} / 1(80 \%$ lymphocytes, $12 \%$ neutrophils, and $8 \%$ monocytes), a protein concentration of $0.56 \mathrm{~g} / \mathrm{l}$ (normal range $0.15-0.45 \mathrm{~g} / \mathrm{l})$, and a glucose concentration of $68 \mathrm{mg} / \mathrm{dl}$ ( $3.77 \mathrm{mmol} / \mathrm{l}$ [normal range $15-$ $45 \mathrm{mg} / \mathrm{l}$ or $0.83-2.50 \mathrm{mmol} / \mathrm{l}]$ ). A CSF $14-3-3$ protein test showed weak immunoreactivity. Evaluations for infectious etiologies, including West Nile virus, Cryptococcus sp., herpes simplex virus (HSV), HIV, malaria, coccidioides, syphilis, enteroviruses, and Borrelia burgdorferi, were unremarkable. The erythrocyte sedimentation rate was mildly elevated at 42 $\mathrm{mm} / \mathrm{h}$ (normal range $0-10 \mathrm{~mm} / \mathrm{h}$ ); titers for antinuclear, antithyroglobulin, and antithyroperoxidase antibodies were negative. A CT scan of the patient's chest and abdomen was unremarkable and an electroencephalogram demonstrated frontal-dominant intermittent rhythmic delta activity. An initial brain MRI scan with fluid-attenuated inversion-recovery (FLAIR) sequences indicated T2-weighted hyperintensities in the medial temporal lobe, including the right caudate nucleus and left insula and involving both white matter and gray matter. Patchy T2 hyperintensities were noted in the external globus pallidus, the left frontal region, and the left putamen. A diagnosis of Creutzfeldt-Jakob disease (CJD) was considered to be likely and the patient's wife contacted the memory disorders clinic for evaluation of the patient.

Upon evaluation at the clinic, the patient was easily distracted, oriented only to name, and had hypophonic speech with decreased output. He displayed little insight and laughed at inappropriate moments. He scored 5 out of 30 on the Mini Mental State Examination (MMSE). He had choppy horizontal smooth pursuit eye movements, full muscle strength, and a broadbased gait. He reported increased sensation to light touch on the left side of his face. He had mildly increased axial tone and a slight intention tremor. His tendon reflexes were normal, and Babinski's and Chaddock's signs were absent.

The patient's past medical illnesses included gout, hypertension, hyperlipidemia, and one episode of a multi-drug-resistant Staphylococcus aureus abscess on his neck 2 years earlier. 
His current medications included irbesartan, allopurinol, atorvastatin, amlodipine, aspirin, and a multivitamin. He traveled to the Philippines regularly; the most recent trip had occurred just before the onset of his first symptoms. Neither the patient nor his wife recalled exposure of the patient to any viral illness, although mosquito bites had been frequent.

The patient was admitted for an expedited evaluation. A repeat CSF analysis revealed a white blood cell count of $12 \times 10^{6} / 1$ (69\% lymphocytes and $28 \%$ monocytes, $2 \%$ neutrophils, $1 \%$ basophils), a protein concentration of $1.37 \mathrm{~g} / \mathrm{l}$, and a glucose concentration of $52 \mathrm{mg} / \mathrm{dl}(2.89$ $\mathrm{mmol} / \mathrm{l}$ ). Evaluations for infectious agents, including bacterial, fungal, and viral sources (St Louis encephalitis, western equine encephalitis, West Nile virus, HSV 1 and 2, varicella-zoster virus, measles, human herpesvirus 6 , influenza $\mathrm{A}$ and $\mathrm{B}$, and adenoviruses), were negative. Results from a CSF polymerase chain reaction (PCR) assay for enterovirus were negative. The CSF 14-3-3 protein assay again showed weak immunoreactivity. Results of a complete paraneoplastic evaluation (NeoComplete ${ }^{\mathrm{TM}}$, Athena Diagnostics, Worcester, MA) and a full body PET scan were negative. A repeat brain MRI scan indicated new FLAIR abnormalities and an increase in the size of previously observed FLAIR abnormalities, as well as new lesions characterized by contrast enhancement (Figure 1). Cerebral angiogram findings were normal.

The patient underwent an open brain biopsy of the anterior left temporal lobe, a region that had shown contrast enhancement on the most recent MRI scan. A routine histological evaluation revealed a diffuse parenchymal gliosis with foci of microglia and lymphocytes, particularly within the perivascular spaces, leptomeninges, and, to a lesser extent, the neuropil (Figure 2). Immunohistochemical studies highlighted a diffuse parenchymal infiltrate of reactive microglial cells and macrophages (CD68), peri-vascular and leptomeningeal accumulations of lymphocytes (leukocyte common antigen), and reactive cells within the parenchyma (glial fibrillary acidic protein), consistent with a meningo-encephalitis. Small and large cerebral arteries, arterioles and capillaries had intact walls, and there was no evidence of mural inflammatory infiltrates, or of necrosis or disruption of the elastic lamina. Stains for spirochetes, fungi, and bacterial organisms were negative. Tissue specimens were sent to the California Encephalitis Project (Richmond, CA) of the California State Health Department. The patient was treated empirically with high-dose intravenous steroids for 5 days, over which time his clinical condition improved.

At the California Encephalitis Project, RNA was extracted from formalin-fixed, paraffinembedded CNS tissue and enterovirus was identified by use of an enterovirus group-specific reverse transcriptase PCR (RT-PCR) assay targeting the $5^{\prime}$ noncoding region of the viral RNA. ${ }^{1}$ Results from PCR assays for HSV 1 and 2, varicella-zoster virus, West Nile virus, and Mycoplasma pneumoniae were negative. The Centers for Disease Control and Prevention (CDC) confirmed the enterovirus finding by use of RNA extraction and enterovirus groupspecific RT-PCR. ${ }^{2,3}$ On the basis of sequence analysis of the $\mathrm{Vp} 1$ region of the virus, the virus was tentatively identified as echovirus 18 . Although the levels of CD4 and CD8 lymphocyte subsets were initially low while the patient was on steroids, a subsequent immunological evaluation completed 4 months later showed normal results, including normal function of both cellular and humoral immunity.

On two occasions - 6 months and 12 months after discharge-the patient was treated with 2 $\mathrm{g} / \mathrm{kg}$ body weight intravenous immunoglobulin in five divided doses. The effectiveness of this treatment was unclear since gradual cognitive improvement continued throughout this period. At 6-month follow-up, the patient's score on the MMSE had improved to 22 out of 30, his wife felt he was at $50 \%$ of pre-illness function, and his weight had stabilized. Further functional improvement was noted at 12-month follow-up, although the patient's MMSE score remained the same. 


\section{DISCUSSION OF DIAGNOSIS}

Enteroviruses are well-known human pathogens in the picornavirus family and include polioviruses, coxsackieviruses, and echoviruses. Acquired predominantly by fecal-oral contamination and, less frequently, by respiratory droplets, there are nearly 70 subtypes of enteroviruses, which are capable of causing a broad spectrum of diseases, typically occurring in children. ${ }^{4}$ Enteroviruses commonly cause CNS infections, accounting for over $75 \%$ of aseptic meningitis cases and up to $27 \%$ of infectious encephalitis cases. 5 The prognosis is generally good, unless the host is less than 2 weeks of age or has compromised humoral immunity, which is often associated with bone marrow transplant or autoimmune disease. ${ }^{4-8}$

Few data exist regarding long-term sequelae in adults with enteroviral meningoencephalitis. ${ }^{6}$ Case reports have described an abrupt onset of enteroviral encephalitis with rapid descent into coma followed by rapid and complete neurological recovery, ${ }^{9-11}$ although long-term cognitive sequelae have also been described. ${ }^{4}$ Focal encephalitis cases involving the temporal lobes and mimicking infections with HSV have been reported. ${ }^{12}$ One case of Coxsackie B encephalitis progressed to seizure, multifocal myoclonus and coma; as with the patient presented in the Case Study, CSF PCR results were negative for enterovirus, and the diagnosis was confirmed by brain biopsy. ${ }^{7}$ During a recent outbreak of enterovirus 71 in children, CSF PCR findings for enterovirus were positive in only $31 \%$ of children with aseptic meningitis, whereas PCR of upper respiratory tract specimens yielded higher sensitivity. ${ }^{13}$

\section{DIFFERENTIAL DIAGNOSIS}

Although rapidly progressive dementia (RPD) syndromes are often considered to be synonymous with CJD, these syndromes have a broad differential diagnosis, which includes auto-immune etiologies, toxic and/or metabolic disturbances, infections, paraneoplastic and other autoimmune processes, and, less frequently, accelerated neurodegenerative disorders. Up to $20 \%$ of CJD cases who are referred to the Memory and Aging Center at the University of California, San Francisco, are determined to have a non-prion disease, and these cases are sometimes treatable. ${ }^{14} \mathrm{~A}$ diagnosis of CJD had been seriously considered in the patient presented here. The mild pleocytosis and the MRI findings substantially decreased this possibility, however, given the presence of both gray and white matter abnormalities that enhanced with gadolinium and appeared in various locations at the second evaluation. Electroencephalogram abnormalities and equivocal results from CSF 14-3-3 protein assays are both less specific than MRI for detection of CJD. ${ }^{14}$ In this patient, the absence of profound CSF pleocytosis resulted in challenges to diagnosis, despite the detection of meningitis at biopsy. Pathologic findings were consistent with meningoencephalitis and ruled out a primary vasculitic process. A microglial response with associated macrophage infiltration, however, can be observed in a plethora of infectious and noninfectious processes and are not characteristic of any one particular infectious agent. These features, along with a predominantly parenchymal lymphocytic infiltrate, suggested many possible etiologies, including a number of viral pathogens but excluding those associated with routinely recognizable inclusions. The CSF PCR findings for enterovirus were negative-as they were in a previous published case $^{7}$ - but the results from this assay should be considered insufficient to exclude enterovirus infection. Although a brain angiogram was conducted, it should be noted that intravascular lymphoma can mimic vasculitis angiographically and, given the high concern for infection, a biopsy would have been indicated regardless of the result of the angiogram.

\section{TREATMENT AND MANAGEMENT}

The accurate diagnosis of RPD syndromes is critical to providing a basis for appropriate planning and an approach to care, although, in this case, no specific diagnostic recommendations emerged. The investigational drug pleconaril, an anti-picornavirus 
compound, has been employed in severe enterovirus infections but could not be obtained in this case. ${ }^{15}$ This patient's clinical course improved substantially following empiric high-dose steroid treatment, and to a lesser degree after subsequent administration of intravenous immunoglobulin. Spontaneous improvement of enterovirus infection has been described; 10 however, in this case the temporal relationship between clinical improvement and administration of high-dose steroids suggests a response to the treatment, although it is possible this was coincidental. To investigate the possibility that the patient's encephalitis was caused by an autoimmune response to the enterovirus, serum (on two occasions) and CSF (on one occasion) were evaluated for the presence of nervous system autoantibodies (laboratory of $\mathbf{J}$ Dalmau, University of Pennsylvania, Philadelphia, PA). No autoantibodies were identified, suggesting that the etiology was not autoimmune.

\section{CONCLUSIONS}

We present a case of enteroviral encephalitis causing an RPD syndrome in an immunocompetent adult. This case highlights an unusual etiology for an adult RPD syndrome and alerts the reader of the broad range of possible conditions that can present with such a syndrome. The clinical approach to this patient was substantially directed by the identification of an enterovirus infection, although specific antiviral treatment approaches were not employed. Diagnostic challenges in this case included a false-negative enterovirus CSF PCR result and an equivocal CSF 14-3-3 protein assay result. Brain MRI findings, however, substantially assisted in excluding CJD. Although not frequently required, advanced techniques such as RNA extraction from and RT-PCR of brain biopsy samples might be necessary to make appropriate diagnoses in some cases.

\section{References}

1. Kares S, et al. Real-time PCR for rapid diagnosis of entero- and rhinovirus infections using LightCycler. J Clin Virol 2004;29:99-104. [PubMed: 14747028]

2. Bhatnagar J, et al. Detection of West Nile virus in formalin-fixed, paraffin-embedded human tissues by RT-PCR: a useful adjunct to conventional tissue-based diagnostic methods. J Clin Virol 2007;38:106-111. [PubMed: 17161650]

3. Thoelen I, et al. Analysis of the serotype and genotype correlation of VP1 and the 5' noncoding region in an epidemiological survey of the human enterovirus B species. J Clin Microbiol 2004;42:963-971. [PubMed: 15004039]

4. Rotbart HA, Hayden FG. Picornavirus infections: a primer for the practitioner. Arch Fam Med 2000;9:913-920. [PubMed: 11031400]

5. Glaser CA, et al. Beyond viruses: clinical profiles and etiologies associated with encephalitis. Clin Infect Dis 2006;43:1565-1577. [PubMed: 17109290]

6. McKinney RE Jr, et al. Chronic enteroviral meningoencephalitis in agammaglobulinemic patients. Rev Infect Dis 1987;9:334-356. [PubMed: 3296100]

7. Berger JR, et al. Persistent Coxsackie B encephalitis: report of a case and review of the literature. J Neurovirol 2006;12:511-516. [PubMed: 17162666]

8. Cree BC, et al. A fatal case of coxsackievirus B4 meningoencephalitis. Arch Neurol 2003;60:107-112. [PubMed: 12533096]

9. Modlin JF, et al. Focal encephalitis with enterovirus infections. Pediatrics 1991;88:841-845. [PubMed: 1896296]

10. Tong CY, et al. Severe encephalitis with rapid recovery. Lancet 1997;349:470. [PubMed: 9040578]

11. Zifroni A, et al. Severe encephalitis with rapid recovery. Lancet 1997;349:1328. [PubMed: 9142093]

12. Liow K, et al. Bilateral hippocampal encephalitis caused by enteroviral infection. Pediatr Neurol 1999;21:836-838. [PubMed: 10593677]

13. Perez-Velez CM, et al. Outbreak of neurologic enterovirus type 71 disease: a diagnostic challenge. Clin Infect Dis 2007;45:950-957. [PubMed: 17879907] 
14. Geschwind MD, et al. Rapidly progressive dementia. Neurol Clin 2007;25:783-807. [PubMed: 17659190]

15. Tormey VJ, et al. SPECT scans for monitoring response to pleconaril therapy in chronic enteroviral meningoencephalitis. J Infect 2003;46:138-140. [PubMed: 12634077] 


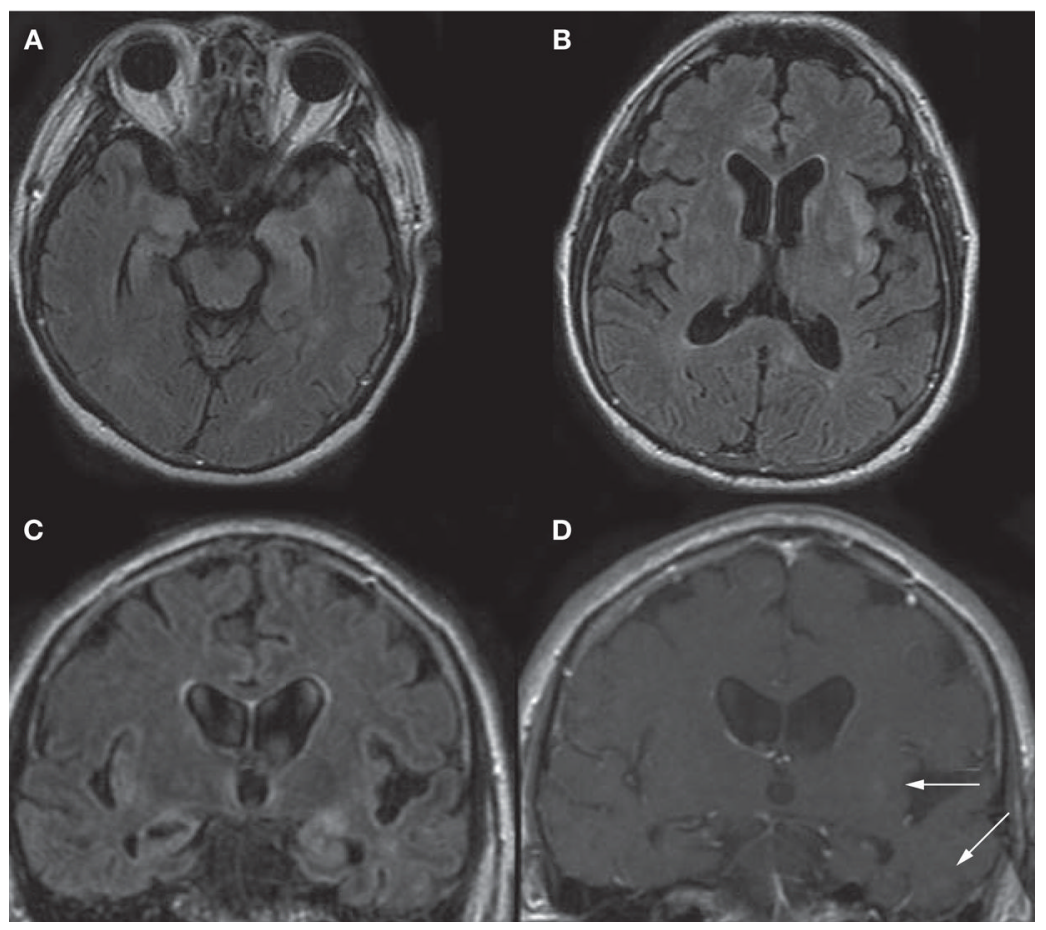

Figure 1.

MRI scans of the patient's brain before surgery. (A-C) A FLAIR sequence demonstrated hyperintensities in gray and white matter, particularly in the medial temporal lobes $(\mathrm{A}, \mathrm{C})$ and in the right anterior cingulate cortex (B), basal ganglia (B) and left insula (B,C). (D) On a T1weighted coronal sequence with contrast, there are small regions of contrast enhancement (arrows) in the left insula and left temporal lobe, corresponding to FLAIR hyperintensities. Abbreviation: FLAIR, fluid-attenuated inversion recovery. 


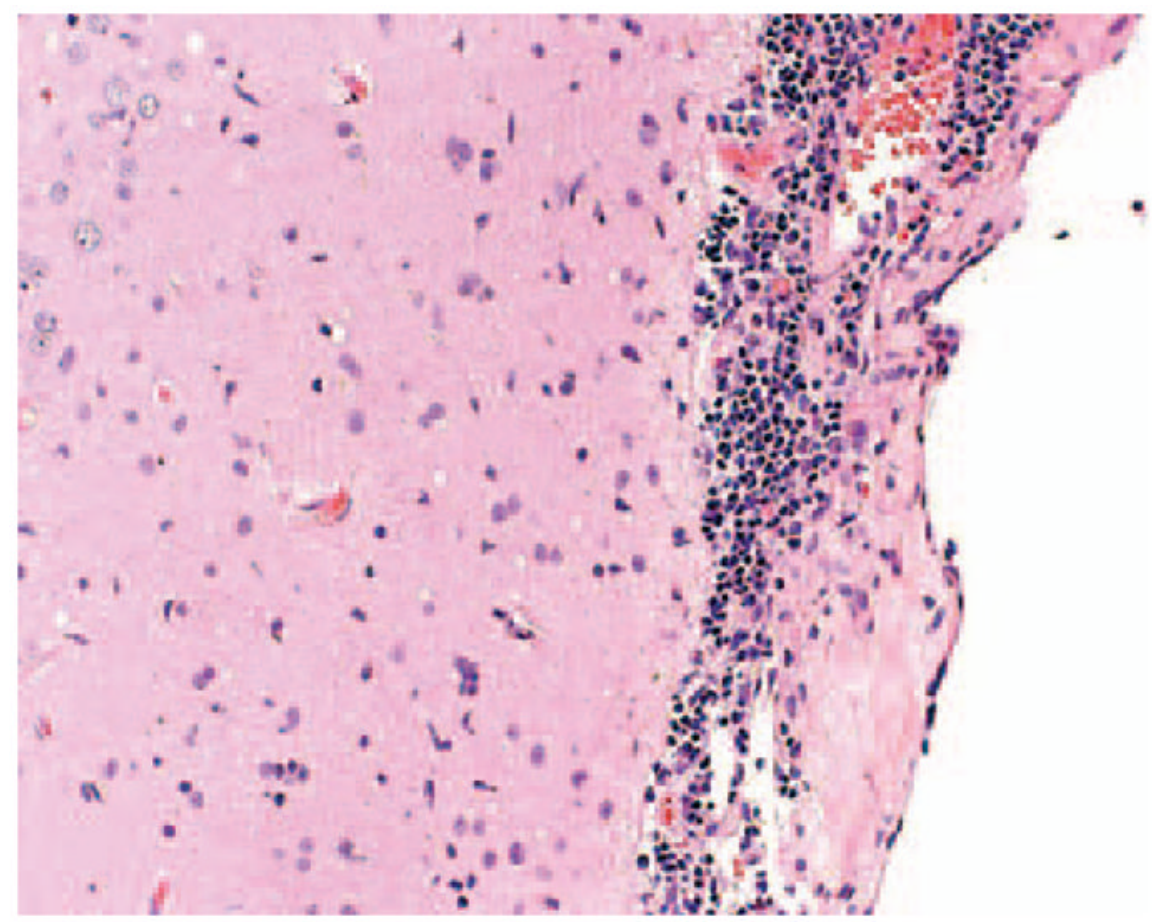

Figure 2.

A brain biopsy specimen stained with hemotoxylin and eosin showed foci of microglia and lymphocytes involving the leptomeninges and neuropil. The specimen also exhibited diffuse parenchymal gliosis (not shown). 